\title{
Blackbody radiation shift in strontium lattice clocks revisited
}

\author{
Ch. Lisdat $\odot$, S. Dörscher $\odot$, I. Nosske $\odot$, and U. Sterr $\odot$ \\ Physikalisch-Technische Bundesanstalt, Bundesallee 100, 38116 Braunschweig, Germany
}

(Received 22 July 2021; accepted 8 November 2021; published 9 December 2021)

\begin{abstract}
We reevaluate the blackbody radiation (BBR) induced ac-Stark shift of the strontium clock transition $(5 s 5 p){ }^{3} P_{0}-\left(5 s^{2}\right){ }^{1} S_{0}$ at $698 \mathrm{~nm}$ used as reference in optical lattice clocks and as secondary representation of the SI unit "second." At room temperature, this frequency shift is on the order of $5 \times 10^{-15}$ of the transition frequency and causes the largest correction in strontium lattice clocks. With the ongoing reduction of measurement uncertainties of optical clocks, an assessment of the approximations made in the evaluation of the BBR shift is advised. Our reevaluation leads to an increase of the BBR correction by $4 \times 10^{-18}$ for clock operation at $300 \mathrm{~K}$, considerably larger than its present uncertainty of $1.6 \times 10^{-18}$. Consistently describing accurately known atomic properties with an atomic structure model, we reduce the fractional uncertainty of the atomic response to $1 \times 10^{-18}$ at $300 \mathrm{~K}$, which allows operating Sr lattice clocks with an uncertainty of $1 \times 10^{-18}$ at room temperature.
\end{abstract}

DOI: 10.1103/PhysRevResearch.3.L042036

Strontium lattice clocks are among the most accurate optical clocks [1-4] and have reported fractional uncertainties of only $2 \times 10^{-18}$ [4]. However, knowledge of atomic response to blackbody radiation (BBR) currently limits their fractional uncertainty to about $1.6 \times 10^{-18}$ when operating at room temperature. The ac-Stark shift $\Delta \nu_{\mathrm{BBR}}(T)$ of the clock reference transition $(5 s 5 p){ }^{3} P_{0}-\left(5 s^{2}\right){ }^{1} S_{0}$ caused by interaction of the strontium atom with the BBR field of the environment [5] typically leads to the largest correction that has to be applied and thus plays an important role in the evaluation of the uncertainty of strontium lattice clocks. In view of a possible redefinition of the SI unit "second" [6], it is paramount to validate candidate clocks at the highest possible level of accuracy and to reconsider the evaluation of the respective uncertainty budgets. Otherwise, undesired jumps in the scale unit of international atomic time can later occur as has happened in the case of caesium clocks by the inclusion of the BBR correction $[7,8]$.

To achieve clock uncertainties of $1 \times 10^{-18}, \Delta \nu_{\mathrm{BBR}}(T)$ has to be determined to two parts in $10^{4}$ in room temperature Sr lattice clocks. Thus, the accurate evaluation of $\Delta \nu_{\mathrm{BBR}}$ has been the subject of several investigations [9-12]. As an alternative to an accurate experimental or theoretical determination of the shift, the shift itself can be reduced by interrogation of the atoms in a cold environment $[13,14]$.

The BBR radiation shift $\Delta v_{\mathrm{BBR}}$ can be measured directly by comparisons of optical clocks at different temperature [13]. However, more accurate determinations currently rely on measurements of the static polarizability difference $\Delta \alpha_{\text {stat }}$ of

Published by the American Physical Society under the terms of the Creative Commons Attribution 4.0 International license. Further distribution of this work must maintain attribution to the author(s) and the published article's title, journal citation, and DOI. the clock states $[9,15]$ and calculations based on the frequencies and strengths of transitions that involve the clock states $[10,11,16]$. The BBR shift can be written as

$$
\begin{aligned}
\Delta \nu_{\mathrm{BBR}}(T) & =-\frac{1}{2 h} \Delta \alpha_{\text {stat }}\left\langle E^{2}\right\rangle_{T}[1+\eta(T)] \\
& =\Delta v^{(\text {stat })}(T)+\Delta v^{(\text {dyn })}(T)
\end{aligned}
$$

with $\left\langle E^{2}\right\rangle_{T} \approx\left(8.319 \mathrm{~V} \mathrm{~cm}^{-1}\right)^{2}(T / 300 \mathrm{~K})^{4}$ the mean squared electric field of the BBR of temperature $T$. The static contribution $\Delta v^{\text {(stat) }}$ to the BBR shift results from the first term of the sum and, for strontium, amounts to $-2.13023(6) \mathrm{Hz}$ at $T=300 \mathrm{~K}$ [9] or $-5 \times 10^{-15}$ in fractional units. The dynamic contribution $\Delta v^{(\mathrm{dyn})}$ originates from $\eta(T)[-148.7(7) \mathrm{mHz}$ or $-3.5 \times 10^{-16}$ at $300 \mathrm{~K}$ [11]]. While $\Delta \alpha_{\text {stat }}$ is known well enough to reduce the uncertainty contribution from $\Delta v^{\text {(stat) }}$ to $1.4 \times 10^{-19}$ at $300 \mathrm{~K}, \Delta v^{(\text {dyn })}$ leads to a ten times larger uncertainty.

$\Delta v^{(\mathrm{dyn})}$ is the difference of the dynamic level shifts $\delta v_{e, g}^{(\mathrm{dyn})}$ of the upper and lower clock state $e$ and $g$, respectively. The $\delta v_{i}^{\text {(dyn) }}$ depend on the atomic Einstein coefficients $A_{k i}$ and the transition frequencies $v_{k i}$ through

$$
\delta v_{i}^{(\mathrm{dyn})}(T)=-\frac{1}{4 \pi^{2}}\left(\frac{k_{B} T}{h}\right)^{3} \sum_{k} \frac{2 J_{k}+1}{2 J_{i}+1} \frac{A_{k i}}{v_{i k}^{3}} G\left(\frac{h v_{i k}}{k_{B} T}\right)
$$

with the Boltzmann and Planck constants $k_{B}$ and $h$ and the angular momenta $J_{i, k}$ of levels $i, k$. The function $G(y)$ is given by the integral [17]

$$
G(y)=\int_{0}^{\infty} \frac{x^{3}}{\exp (x)-1}\left(\frac{2 y}{y^{2}-x^{2}}-\frac{2}{y}\right) d x
$$


Often, this integral is approximated by a Taylor expansion in $1 / y \propto T$ up to order $n[5,10,16]:$

$$
G(y) \approx 2 \sum_{k=3}^{n} \frac{(-1)^{k-1}}{4 k} \frac{(2 \pi)^{2 k}}{y^{2 k-3}} B_{2 k} \equiv G_{n}(y)
$$

with the Bernoulli numbers $B_{2 k}$. This series leads to the known expansion of the dynamic contribution in $T^{6}, T^{8}$, and higher orders. However, the convergence radius of the Taylor expansion of $G$ in $1 / y$ is zero, as shown analytically in Appendix A.

In the particular case of the strontium atom, the $(5 s 5 p){ }^{3} P_{0}$ and $(5 s 4 d){ }^{3} D_{1}$ states are coupled by a low energy transition at about $2.6 \mu \mathrm{m}$. The contribution from this transition dominates the dynamic contributions $\delta v_{e}^{(\mathrm{dyn})}$ and $\Delta v^{(\mathrm{dyn})}$. Its approximation requires more terms in Eq. (4) than the other transitions. In the most recent calculation that is currently the basis for correcting all Sr lattice clocks, Safronova et al. [10] used three terms up to $T^{10}$ for this transition. Approximating Eq. (3) to this order was sufficiently accurate at the time of the publication. Given the improved knowledge of Einstein coefficients [11] and the overall improvements on strontium lattice clocks, this approximation has to be reconsidered.

We find that the difference between the exact values of $G$ at $T=300 \mathrm{~K}$ as derived from Eq. (3) and its approximation with three terms [Eq. (4)] amounts to $1.4 \%$ for the $2.6 \mu \mathrm{m}{ }^{3} P_{0}$ and ${ }^{3} D_{1}$ transition in strontium. Since this transition is responsible for $98 \%$ of $\delta v_{e}^{(\mathrm{dyn})}$ [10], the presently accepted dynamic contribution $\Delta v^{(\mathrm{dyn})}(300 \mathrm{~K})$ of $-148.7 \mathrm{mHz}$ [11] must be reduced by about the same fraction. In consequence, the frequency of a strontium lattice clock operated at room temperature and corrected for the BBR shift using the value from [11] is currently low by $2.0 \mathrm{mHz}$, which amounts to $4.7 \times 10^{-18}$ in fractional units. This difference exceeds the uncertainty of the most accurate strontium lattice clocks $[4,11]$ by more than $2 \sigma$.

Extending the power series in Eq. (4) does not improve the result in the case of strontium because the expansion does not allow for a sufficiently accurate approximation of Eq. (3) at $300 \mathrm{~K}$ for the $2.6 \mu \mathrm{m}$ transition. $\left|1-G_{n} / G\right|$ is plotted in Fig. 1 for this transition as well as the corresponding transition $(6 s 5 d){ }^{3} D_{1}-(6 s 6 p){ }^{3} P_{0}$ at $1.4 \mu \mathrm{m}$ in $\mathrm{Yb}$. We conclude that a Taylor expansion of $G$ is not viable for the $2.6 \mu \mathrm{m}$ transition in strontium at $T=300 \mathrm{~K}$. For the other transitions, an approximation of $G(y)$ with low $n$ is sufficiently accurate in terms of the present clock uncertainty. Numerically integrated values of $G$ of the $2.6 \mu \mathrm{m}$ transition for different temperatures are provided as open data [18].

When we calculate the dynamic contribution $\Delta v^{(\mathrm{dyn})}$ from the contributions given in Ref. [10] but replace the Taylor expansion by the accurate numerical value of the integral for the $(5 s 4 d){ }^{3} D_{1}-(5 s 5 p){ }^{3} P_{0}$ contribution from Eqs. (2) and (3) using the Einstein coefficient and branching ratios for the decay channels of the ${ }^{3} D_{1}$ level [11], we find $\Delta v^{(\text {dyn })}=$ $-150.7 \mathrm{mHz}$. The uncertainty of this value is dominated by the uncertainty of the ${ }^{3} D_{1}$ lifetime and thus equal to the uncertainty of $0.7 \mathrm{mHz}$ stated in Ref. [11]. Note that the accurate variation of $\Delta \nu_{\mathrm{BBR}}$ with temperature $T$ can be derived similarly to the approach in [12] from the parameters given in [10],

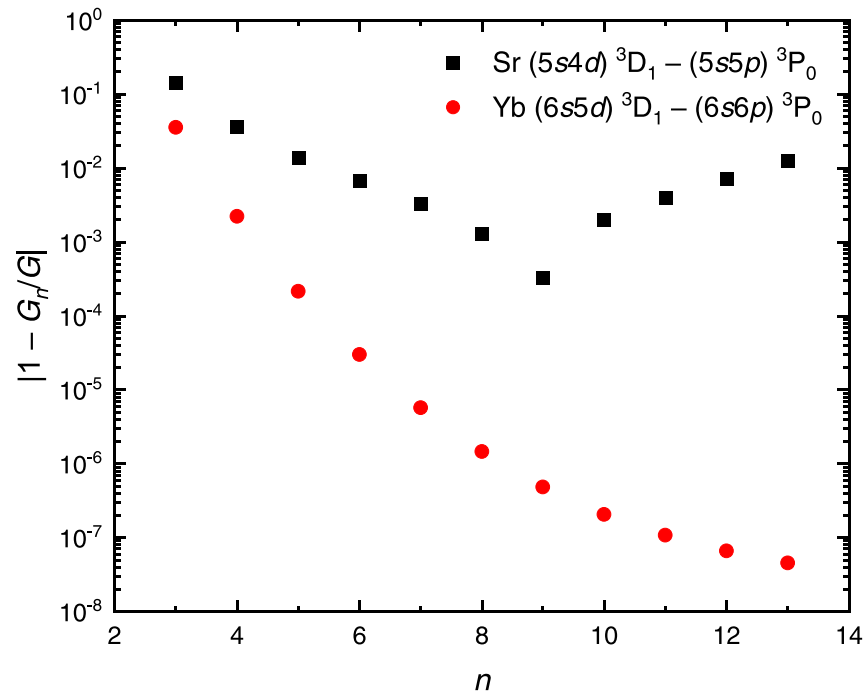

FIG. 1. Convergence of the Taylor expansion of $G(y)$ [Eq. (4)] for $y=18.4$ and 34.5 , corresponding to $T=300 \mathrm{~K}$ and the energetically lowest transitions ${ }^{3} P_{0}-{ }^{3} D_{1}$ in strontium (squares) and ytterbium (dots), respectively. Note that $\left(1-G_{n} / G\right)$ changes its sign for the case of strontium.

Eq. (2) with $v$ and $A$ of the transition ${ }^{3} D_{1}-{ }^{3} P_{0}$, and the tabulated values for $G$ [18].

However, this approach does not make the best use of the experimental information we have on the strontium atom and their interconnection provided by the atomic structure. As mentioned above, an accurate value for the static polarizability difference $\Delta \alpha_{\text {stat }}$ of the states $(5 s 5 p){ }^{3} P_{0}$ and $\left(5 s^{2}\right){ }^{1} S_{0}$ is available [9]; the static $\left(5 s^{2}\right){ }^{1} S_{0}$ polarizability is also known from experiment $[24]$ and theory $[5,25,26]$ (an overview is found in [27]). While Porsev and Derevianko [5] include experimental information, Refs. $[25,26]$ rely on theory only. The latter results have a small uncertainty but deviate considerably. Measurements of the scalar ac-Stark shift cancellation ("magic") wavelength at $813 \mathrm{~nm}[28,29]$ and $390 \mathrm{~nm}$ [30] exist as well as a determination of the derivative of the dynamic polarizability difference of the clock states at $813 \mathrm{~nm}$ [31]. The tune-out frequency near the $689 \mathrm{~nm}$ intercombination line, at which the ground state polarizability vanishes, and the polarizability of the ${ }^{3} P_{0}$ state at this frequency have been reported [32]. In addition, the differential polarizability of the clock reference transition at $698 \mathrm{~nm}$ has been measured, but the values differ strongly [33-35]. Furthermore, ThomasReiche-Kuhn sum rules introduce additional constraints [36]. These quantities are all closely related to the atomic transition frequencies $v_{i k}$ and Einstein coefficients $A_{k i}$ and can be calculated in a similar way as $\delta v_{i}^{(\mathrm{dyn})}$ using the dynamic polarizabilities $\alpha(v)$ of the clock states

$$
\alpha_{i}(v)=\alpha_{i}^{\text {core }}+\frac{\epsilon_{0} c^{3}}{(2 \pi)^{3}} \sum_{k} \frac{2 J_{k}+1}{2 J_{i}+1} \frac{A_{k i}}{v_{i k}^{2}\left(v_{i k}^{2}-v^{2}\right)} .
$$

We use the transitions listed in Tables I and II to calculate $\Delta v_{\mathrm{BBR}}$ and the above-mentioned quantities and adjust selected $A_{k i}$ coefficients to best describe the experimental data. Additionally, we add ionic core polarizabilities $\alpha_{i}^{\text {core }}$ for each 
TABLE I. Lines connecting to the $\left(5 s^{2}\right){ }^{1} S_{0}$ level. Accurate transition energies are derived from atomic data bases [23]. Lines used in the fit are marked by F (for details, see the main text, Appendix B, and open data [18]).

\begin{tabular}{lccc}
\hline \hline Upper level & Wavelength & $A_{k i}\left(\mathrm{~s}^{-1}\right)$ & Reference \\
\hline$(5 s 5 p){ }^{3} P_{1}$ & $689 \mathrm{~nm}$ & $4.699(7) \times 10^{4}$ & {$[11]$} \\
$(5 s 5 p){ }^{1} P_{1}$ & $461 \mathrm{~nm}$ & $1.9001(14) \times 10^{8}$ & {$[19]$} \\
$(5 s 6 p){ }^{1} P_{1}$ & $293 \mathrm{~nm}$ & $1.9(3) \times 10^{6}$ & {$[20]$} \\
$(5 s 7 p){ }^{1} P_{1}$ & $257 \mathrm{~nm}$ & $5.3(6) \times 10^{6}$ & {$[20]$} \\
$(5 s 8 p){ }^{1} P_{1}$ & $236 \mathrm{~nm}$ & $1.8(3) \times 10^{7}$ & {$[20]$} \\
$(5 s 9 p){ }^{1} P_{1}$ & $231 \mathrm{~nm}$ & $1.15(17) \times 10^{7}$ & {$[20]$} \\
$(5 s 10 p){ }^{1} P_{1}$ & $228 \mathrm{~nm}$ & $6.6(8) \times 10^{6}$ & {$[20]$} \\
$(5 s 11 p){ }^{1} P_{1}$ & $225 \mathrm{~nm}$ & $3.7(4) \times 10^{6}$ & {$[20]$} \\
$(5 s 12 p){ }^{1} P_{1}$ & $224 \mathrm{~nm}$ & $2.4(3) \times 10^{6}$ & {$[20]$} \\
$(5 s 13 p){ }^{1} P_{1}$ & $223 \mathrm{~nm}$ & $1.57(19) \times 10^{6}$ & {$[20]$} \\
$(5 s 14 p){ }^{1} P_{1}$ & $222 \mathrm{~nm}$ & $8.5(10) \times 10^{5}$ & {$[20]$} \\
$(5 s 15 p){ }^{1} P_{1}$ & $221 \mathrm{~nm}$ & $1.7(3) \times 10^{7}$ & Estimated \\
$(4 d 5 p){ }^{1} P_{1}$ & $243 \mathrm{~nm}$ & $\approx 1.65 \times 10^{8}$ & from [21,22] \\
Rydberg and & $\approx 218 \mathrm{~nm}$ & & $\mathrm{~F}$ \\
cont. ${ }^{1} P_{1}$ & & & \\
\hline \hline
\end{tabular}

state including corrections due to the presence of the two valence electrons [10]. The source code is derived from the one used in Ref. [9]. Where available [10,11,19,20], we use data for the fine-structure transitions. Otherwise [39,40], we calculate the required $A_{k i}$ assuming $L S$ coupling and including a scaling of the Einstein coefficients by the ratio of the actual and the fine-structure-free transition frequencies to the power of 3 .

During the fit, the variation of the Einstein coefficients of the lower levels $(5 s 5 p){ }^{3} P_{1},(5 s 5 p){ }^{1} P_{1},(5 s 6 s){ }^{3} S_{1}$, and $(5 s 4 d){ }^{3} D_{1}$ were constrained by including their uncertainties in the fit routine. For the higher lying levels, the fitted $A_{k i}$ should be interpreted as effective adjustment of the manifold of neighboring levels. For the ${ }^{1} S_{0}$ static polarizability, we chose the experimental value [24] because results from Refs. [25,26] are discrepant and Ref. [5] heavily relies on the experimental determination of the lifetime of the lowest ${ }^{1} P_{1}$ state, which is included independently in our analysis. The polarizability difference at the clock transition [33-35] is not included in the fit because the observations are in disagreement, too. The fit constraints are summarized in Table III and visualized in Fig. 2. We believe that this fit procedure most efficiently combines the available information and the constraints imposed by the atomic structure to minimize the uncertainty in the determination of $\Delta v^{\text {(dyn) }}$.

Running the fit with all constraining data in Table III ended resulted in a $\chi^{2} \approx 37$ (see Appendix $\mathrm{B}$ for detailed fit results), indicating that the data set is not consistent. Dropping the Thomas-Reiche-Kuhn sums from the list of constraints reduces the $\chi^{2}$ to seven. Similar reductions are observed if data from Ref. [32] is omitted or, in addition to the Thomas-Reiche-Kuhn sum, also the blue magic wavelength is excluded. In the latter two fits, we reduced the number of fit parameters to six and seven, respectively (Appendix B). The

TABLE II. Lines connecting to the $(5 s 5 p){ }^{3} P_{0}$ level. Accurate transition energies are derived from atomic data bases [23]. Lines used in the fit are marked by F (for details, see the main text, Appendix B, and open data [18]). If no fine-structure-resolved data are available (e), the respective $A_{k i}$ are estimated (see text).

\begin{tabular}{|c|c|c|c|c|}
\hline Upper level & Wavelength & $A_{k i}\left(\mathrm{~s}^{-1}\right)$ & \multicolumn{2}{|c|}{ Reference } \\
\hline$(5 s 6 s){ }^{3} S_{1}$ & $679 \mathrm{~nm}$ & $7.7(4) \times 10^{6}$ & {$[37,38]$} & $\mathrm{F}$ \\
\hline$(5 s 7 s){ }^{3} S_{1}$ & $433 \mathrm{~nm}$ & $3.1(2) \times 10^{6}$ & {$[20]$} & \\
\hline$(5 s 8 s){ }^{3} S_{1}$ & $378 \mathrm{~nm}$ & $9.34 \times 10^{5}$ & [39] & $\mathrm{e}$ \\
\hline$(5 s 9 s){ }^{3} S_{1}$ & $355 \mathrm{~nm}$ & $5.14 \times 10^{5}$ & [39] & $\mathrm{e}$ \\
\hline$(5 s 10 s){ }^{3} S_{1}$ & $344 \mathrm{~nm}$ & $3.14 \times 10^{5}$ & [39] & $\mathrm{e}$ \\
\hline$\left(5 p^{2}\right){ }^{3} P_{1}$ & $474 \mathrm{~nm}$ & $3.9(3) \times 10^{7}$ & {$[20]$} & \\
\hline$\left(4 d^{2}\right){ }^{3} P_{1}$ & $330 \mathrm{~nm}$ & $4.93 \times 10^{7}$ & [40] & $\mathrm{e}$ \\
\hline$(5 s 4 d){ }^{3} D_{1}$ & $2603 \mathrm{~nm}$ & $2.731(13) \times 10^{5}$ & {$[10,11]$} & $\mathrm{F}$ \\
\hline$(5 s 5 d){ }^{3} D_{1}$ & $483 \mathrm{~nm}$ & $3.3(2) \times 10^{7}$ & {$[20]$} & $\mathrm{F}$ \\
\hline$(5 s 6 d){ }^{3} D_{1}$ & $394 \mathrm{~nm}$ & $1.43 \times 10^{7}$ & [41] & $\mathrm{F}$ \\
\hline$(5 s 7 d){ }^{3} D_{1}$ & $363 \mathrm{~nm}$ & $8.02 \times 10^{6}$ & [39] & $\mathrm{e}$ \\
\hline$(5 s 8 d){ }^{3} D_{1}$ & $348 \mathrm{~nm}$ & $4.81 \times 10^{6}$ & [39] & $\mathrm{e}$ \\
\hline$(5 s 9 d){ }^{3} D_{1}$ & $339 \mathrm{~nm}$ & $3.11 \times 10^{6}$ & [39] & $\mathrm{e}$ \\
\hline Rydberg and cont. ${ }^{3} S_{1}$ & $\approx 316 \mathrm{~nm}$ & $1.8 \times 10^{6}$ & [21] & \\
\hline Rydberg and cont. ${ }^{3} D_{1}$ & $\approx 316 \mathrm{~nm}$ & $4.2 \times 10^{7}$ & [21] & $\mathrm{F}$ \\
\hline
\end{tabular}


TABLE III. Constraints used for the improved determination of the dynamic BBR shift. $E_{r}=h^{2} /\left(2 m \lambda^{2}\right)$ is the photon recoil energy at the magic lattice wavelength $\lambda$ for an atom of mass $m$.

\begin{tabular}{|c|c|c|}
\hline Property & Literature value & Reference \\
\hline$A\left(5 s 5 p{ }^{1} P_{1} \rightarrow 5 s^{2}{ }^{1} S_{0}\right)$ at $461 \mathrm{~nm}$ & $1.9001(14) \times 10^{8} \mathrm{~s}^{-1}$ & [19] \\
\hline$A\left(5 s 5 p{ }^{3} P_{1} \rightarrow 5 s^{2}{ }^{1} S_{0}\right)$ at $689 \mathrm{~nm}$ & $4.699(7) \times 10^{4} \mathrm{~s}^{-1}$ & [11] \\
\hline$A\left(5 s 4 d{ }^{3} D_{1} \rightarrow 5 s 5 p{ }^{3} P_{0}\right)$ at $2.6 \mu \mathrm{m}$ & $2.731(13) \times 10^{5} \mathrm{~s}^{-1}$ & {$[10,11]$} \\
\hline$A\left(5 s 6 s{ }^{3} S_{1} \rightarrow 5 s 5 p^{3} P_{0}\right)$ at $679 \mathrm{~nm}$ & $7.7(4) \times 10^{6} \mathrm{~s}^{-1}$ & {$[37,38]$} \\
\hline$\alpha\left(5 s^{2}{ }^{1} S_{0}, v \rightarrow 0\right)$ & $3.07(24) \times 10^{-39} \mathrm{C} \mathrm{m}^{2} / \mathrm{V}$ & [24] \\
\hline$\Delta \alpha\left(5 s 5 p{ }^{3} P_{0}-5 s^{2}{ }^{1} S_{0}, v \rightarrow 0\right)$ & $4.07873(11) \times 10^{-39} \mathrm{C} \mathrm{m}^{2} / \mathrm{V}$ & [9] \\
\hline Magic frequency near $813 \mathrm{~nm}$ & $368554.725(3) \mathrm{GHz}$ & {$[28]$} \\
\hline$d \Delta \alpha / d v$ at $813 \mathrm{~nm}$ magic frequency & $1.735(13) \times 10^{-5} \mathrm{~Hz} /\left(E_{\mathrm{r}} \mathrm{MHz}\right)$ & [31] \\
\hline Magic frequency near $390 \mathrm{~nm}$ & $768917(18) \mathrm{GHz}$ & [30] \\
\hline Tune-out frequency $v_{\text {to }}$ near $689 \mathrm{~nm}$ & $434972.130(10) \mathrm{GHz}$ & [32] \\
\hline$\alpha\left(5 s 5 p^{3} P_{0}, v=v_{\mathrm{to}}\right)$ & $2.564(13) \times 10^{-38} \mathrm{C} \mathrm{m}^{2} / \mathrm{V}$ & {$[32]$} \\
\hline $5 s^{2}{ }^{1} S_{0}$ Thomas-Reiche-Kuhn sum & $2.0(1)$ & [36] \\
\hline $5 s 5 p{ }^{3} P_{0}$ Thomas-Reiche-Kuhn sum & $2.0(1)$ & [36] \\
\hline
\end{tabular}

variation of the fitted $A_{k i}$ remains in an acceptable range in all fits.

To determine the uncertainty of $\Delta v^{(\mathrm{dyn})}$ in each fit, we vary input parameters constraining the fit within their uncertainties by a Monte Carlo algorithm and rerun the fit. All parameters are treated as uncorrelated. The results from all four fits agree well within their uncertainties (Appendix B). Since we cannot identify a preferable fit, we calculate the weighted mean with the reciprocal squared uncertainties as weights. The uncertainty is calculated under the assumption of fully correlated data [42], i.e., as weighted mean using the same weights as above. We find $\Delta v^{(\mathrm{dyn})}(300 \mathrm{~K})=$ $-150.51(43) \mathrm{mHz}$, which is consistent with our result above but at an uncertainty that is reduced by a factor of 1.6. The

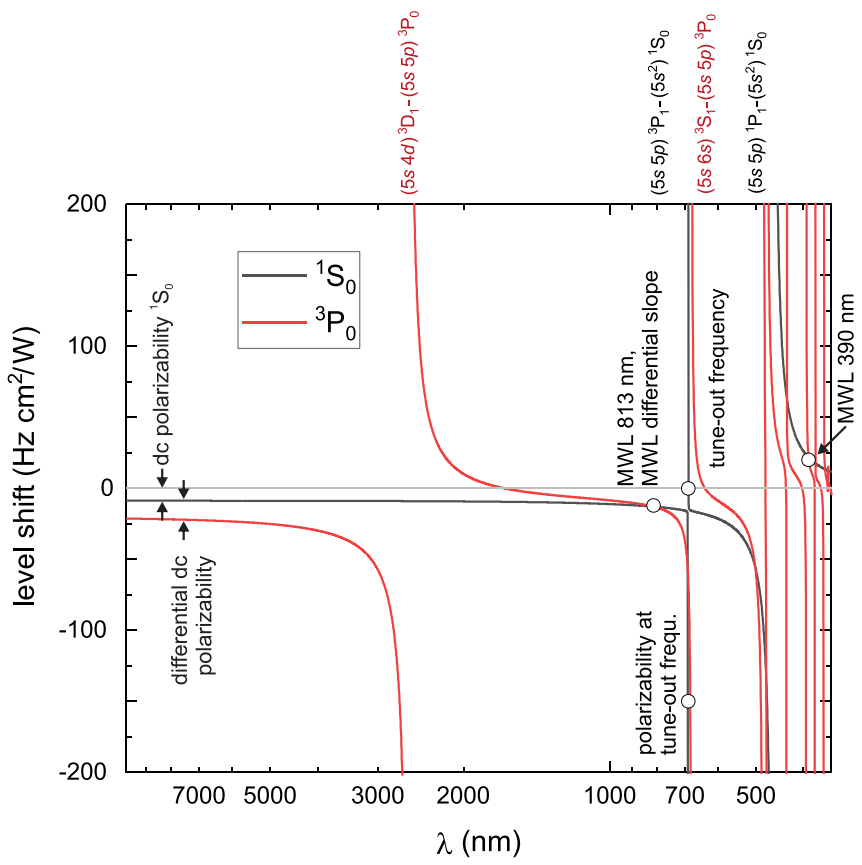

FIG. 2. Frequency-dependent ac-Stark shift of the clock states $\left(5 s^{2}\right){ }^{1} S_{0}$ and $(5 s 5 p){ }^{3} P_{0}$. Annotations indicate where experimental data constrain the fit. MWL: magic wavelength. uncertainty is also below the previously reported uncertainty of $\Delta v^{(\text {dyn })}(300 \mathrm{~K})=-148.7(7) \mathrm{mHz}[11,12]$. Both values do not agree within their uncertainty for the reasons discussed earlier. The difference between both corrections is larger than the lowest uncertainties reported for strontium lattice clocks $[4,11]$. Our reevaluation agrees with the experimental investigation of the BBR shift using cryogenic strontium lattice clocks [13] within the reported uncertainties. The authors report $\Delta v^{(\text {dyn })}(300 \mathrm{~K})=-148.0(26) \mathrm{mHz}$. Our result is slightly discrepant with our earlier work [9], which resulted in $\Delta v^{\text {(dyn) }}(300 \mathrm{~K})=-147.6(23) \mathrm{mHz}$. At the time, the accurate measurement of the $5 s 4 d^{3} D_{1}$ Einstein coefficient [10,11] was not yet available. Its currently known value is about $2 \%$ larger than the fit result in [9], which explains the change of $\Delta v^{(\mathrm{dyn})}$.

The dynamic contribution $\Delta \nu_{\mathrm{BBR}}$ can in principle be calculated for arbitrary temperatures with parameters as given in Tables I-III and Eq. (2). However, this is inconvenient in practice. Moreover, it is difficult to give a single set of parameters that reproduces the averaged dynamic contribution we have determined. Therefore, we calculate the temperature dependence of $\Delta v_{\mathrm{BBR}}$ for the four fit results and determine their weighted average. The result is provided as open data [18]. For convenience in practical use, we fit these data in the temperature range from 50 to $350 \mathrm{~K}$ by the polynomial

$$
\Delta v^{(\text {dyn })} \approx \eta_{6}\left(\frac{T}{300 \mathrm{~K}}\right)^{6}+\eta_{8}\left(\frac{T}{300 \mathrm{~K}}\right)^{8}+\eta_{10}\left(\frac{T}{300 \mathrm{~K}}\right)^{10}
$$

which covers the likely operation range of strontium lattice clocks. With the coefficients

$$
\begin{aligned}
\eta_{6} & =-0.12998 \mathrm{~Hz}, \\
\eta_{8} & =-0.01211 \mathrm{~Hz}, \\
\eta_{10} & =-0.00844 \mathrm{~Hz},
\end{aligned}
$$

it reproduces the input $\Delta v^{(\mathrm{dyn})}(T)$ with residuals smaller than the uncertainty of $\Delta v_{\mathrm{BBR}}$ (Fig. 3).

In conclusion, we have demonstrated that approximating the dynamic BBR correction in strontium lattice clocks by its Taylor expansion produces errors that are beyond the required level of accuracy. The resulting dynamic BBR correction 


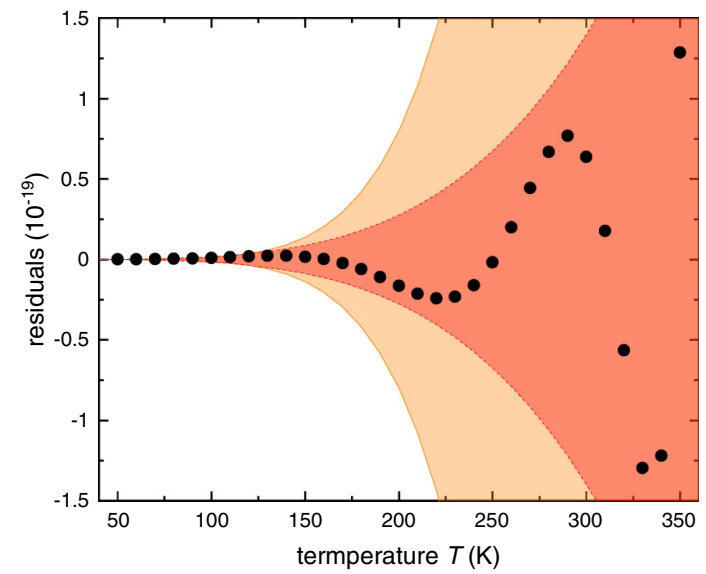

FIG. 3. Residuals of the polynomial Eq. (6) fitted to the calculated $\Delta v^{(\mathrm{dyn})}(T)$ (dots). The light-shaded region shows the $1 \sigma$-confidence interval of $\Delta v^{(\mathrm{dyn})}$, and the darker area that of $\Delta v^{\text {(stat) }}$.

differs from the correct value by twice the uncertainty of the most accurate strontium lattice clocks. With comprehensive modeling of the spectroscopic data and structure of strontium, we can lower the uncertainty of $\Delta v^{(\mathrm{dyn})}$ and thus reduce the achievable uncertainty of room temperature strontium lattice clocks to $1 \times 10^{-18}$. For practical use, we provide a simple interpolation formula to determine the BBR correction in a temperature range from 50 to $350 \mathrm{~K}$.

In order to further reduce the uncertainty of $\Delta v^{(\mathrm{dyn})}(T)$, a more consistent description of the atomic structure and the various observations is called for. Additional accurate determinations of Einstein coefficients and other atomic parameters will be necessary. Lastly, higher-order multipole transitionsspecifically, magnetic-dipole $(M 1)$ transitions-contribute to the BBR shift at the level of $6 \times 10^{-20}$ [5] and will need to be taken into account if the uncertainty is reduced by about an order of magnitude from the present uncertainty.

Note added. Recently, Park et al. [43] report an experimental determination of the ratio $\alpha\left(5 s^{2}{ }^{1} S_{0}\right) / \alpha\left(5 s 5 p{ }^{3} P_{0}\right)=$ $1.1885(10)$ at $914.332 \mathrm{~nm}$ and a theoretical estimate of $1.18(1)$. From our fits, we find a value of 1.179 , which is in reasonable agreement with their results.

We acknowledge support by the Project 18SIB05 ROCIT, which has received funding from the EMPIR programme cofinanced by the Participating States and from the European Union's Horizon 2020 Research and Innovation Programme, and by the Deutsche Forschungsgemeinschaft (DFG, German Research Foundation) under Germany's Excellence StrategyEXC-2123 QuantumFrontiers, Project-ID 390837967; SFB 1464 TerraQ, Project-ID 434617780, within Project A04; and SFB 1227 DQ-mat, Project-ID 274200144, within Project B02. This work was partially supported by the Max PlanckRIKEN-PTB Center for Time, Constants and Fundamental Symmetries funded equally by the three partners.

\section{APPENDIX A: CONVERGENCE RADIUS}

In Eq. (4) of the main text the function $G(y)$ is approximated by a series $G_{\infty}$. This series can be written as a power series in $z=1 / y^{2}$ :

$$
G_{\infty}(y)=2 z^{-3 / 2} \sum_{k=3}^{\infty} \frac{(-1)^{k-1}(2 \pi)^{2 k}}{4 k} B_{2 k} z^{k} .
$$

TABLE IV. Summary of the fits and derived parameters to determine $\Delta v^{(\mathrm{dyn})}$. When the literature value of an atomic property was used to constrain the fit by the given uncertainty, we report the resulting deviation from the literature value in units of that uncertainty $\sigma$. When these values were fitted without constraints, we report the fitted value. Dashes mark parameters that were not used as fit parameters. $E_{r}=h^{2} /\left(2 m \lambda^{2}\right)$ is the photon recoil energy at the magic lattice wavelength $\lambda$ for an atom of mass $m$.

\begin{tabular}{|c|c|c|c|c|c|c|}
\hline Property & Fit 1 & Fit 2 & Fit 3 & Fit 4 & Literature value & ref. \\
\hline Dynamic contribution $\Delta v^{(\mathrm{dyn})}(300 \mathrm{~K})$ & $-150.61(44) \mathrm{mHz}$ & $-150.33(51) \mathrm{mHz}$ & $-150.63(36) \mathrm{mHz}$ & $-150.32(51) \mathrm{mHz}$ & $-148.7(7) \mathrm{mHz}$ & [11] \\
\hline$\chi^{2}$ of fit & 36.6 & 7.0 & 8.5 & 7.0 & & \\
\hline$A\left(5 s 5 p{ }^{1} P_{1} \rightarrow 5 s^{2}{ }^{1} S_{0}\right)$ at $461 \mathrm{~nm}$ & $0.471 \sigma$ & $-0.01 \sigma$ & $0.01 \sigma$ & $-0.01 \sigma$ & $1.9001(14) \times 10^{8} \mathrm{~s}^{-1}$ & [19] \\
\hline$A\left(5 s 5 p{ }^{3} P_{1} \rightarrow 5 s^{2}{ }^{1} S_{0}\right)$ at $689 \mathrm{~nm}$ & $0.795 \sigma$ & $0.177 \sigma$ & - & $0.181 \sigma$ & $4.699(7) \times 10^{4} \mathrm{~s}^{-1}$ & {$[11]$} \\
\hline$A_{\text {eff }}\left({ }^{1} P_{1}\right.$ continuum $\left.\rightarrow 5 s^{2}{ }^{1} S_{0}\right)$ & $2.42 \times 10^{8} \mathrm{~s}^{-1}$ & $2.53 \times 10^{8} \mathrm{~s}^{-1}$ & $4.76 \times 10^{8} \mathrm{~s}^{-1}$ & $2.53 \times 10^{8} \mathrm{~s}^{-1}$ & $1.65 \times 10^{8} \mathrm{~s}^{-1}$ & {$[21,22]$} \\
\hline$A\left(5 s 4 d{ }^{3} D_{1} \rightarrow 5 s 5 p{ }^{3} P_{0}\right)$ at $2.6 \mu \mathrm{m}$ & $-0.554 \sigma$ & $-0.938 \sigma$ & $-0.644 \sigma$ & $-0.950 \sigma$ & $2.731(13) \times 10^{5} \mathrm{~s}^{-1}$ & {$[10,11]$} \\
\hline$A\left(5 s 6 s{ }^{3} S_{1} \rightarrow 5 s 5 p^{3} P_{0}\right)$ at $679 \mathrm{~nm}$ & $1.607 \sigma$ & $1.671 \sigma$ & $-0.170 \sigma$ & $1.669 \sigma$ & $7.7(4) \times 10^{6} \mathrm{~s}^{-1}$ & {$[37,38]$} \\
\hline$A\left(5 s 5 d{ }^{3} D_{1} \rightarrow 5 s 5 p{ }^{3} P_{0}\right)$ at $483 \mathrm{~nm}$ & $2.89 \times 10^{7} \mathrm{~s}^{-1}$ & $2.66 \times 10^{7} \mathrm{~s}^{-1}$ & $4.76 \times 10^{7} \mathrm{~s}^{-1}$ & $2.73 \times 10^{7} \mathrm{~s}^{-1}$ & $3.3(2) \times 10^{7} \mathrm{~s}^{-1}$ & {$[20]$} \\
\hline$A\left(5 s 6 d^{3} \mathrm{D}_{1} \rightarrow 5 s 5 p^{3} P_{0}\right)$ at $394 \mathrm{~nm}$ & $1.86 \times 10^{7} \mathrm{~s}^{-1}$ & $1.92 \times 10^{7} \mathrm{~s}^{-1}$ & - & - & $1.43 \times 10^{7} \mathrm{~s}^{-1}$ & [20] \\
\hline$A_{\text {eff }}\left({ }^{3} D_{1}\right.$ continuum $\left.\rightarrow{ }^{3} P_{0}\right)$ & $1.47 \times 10^{8} \mathrm{~s}^{-1}$ & $1.62 \times 10^{8} \mathrm{~s}^{-1}$ & $2.75 \times 10^{8} \mathrm{~s}^{-1}$ & $1.70 \times 10^{8} \mathrm{~s}^{-1}$ & $4.2 \times 10^{7} \mathrm{~s}^{-1}$ & {$[21,22]$} \\
\hline$\alpha\left(5 s^{2}{ }^{1} S_{0}, v \rightarrow 0\right)$ & $1.47 \sigma$ & $1.50 \sigma$ & $0.83 \sigma$ & $1.50 \sigma$ & $3.07(24) \times 10^{-39} \mathrm{C} \mathrm{m}^{2} / \mathrm{V}$ & {$[24]$} \\
\hline$\Delta \alpha\left(5 s 5 p{ }^{3} P_{0}-5 s^{2}{ }^{1} S_{0}, v \rightarrow 0\right)$ & $-2.19 \times 10^{-3} \sigma$ & $-4.66 \times 10^{-3} \sigma$ & $-3.70 \times 10^{-3} \sigma$ & $-4.72 \times 10^{-3} \sigma$ & $4.07873(11) \times 10^{-39} \mathrm{C} \mathrm{m}^{2} / \mathrm{V}$ & {$[9]$} \\
\hline Magic frequency near $813 \mathrm{~nm}$ & $-1 \times 10^{-6} \sigma$ & $2 \times 10^{-6} \sigma$ & $1 \times 10^{-6} \sigma$ & $2 \times 10^{-6} \sigma$ & $368554.725(3) \mathrm{GHz}$ & {$[28]$} \\
\hline$d \Delta \alpha / d v$ at $813 \mathrm{~nm}$ magic frequency & $1.31 \sigma$ & $0.79 \sigma$ & $0.04 \sigma$ & $0.80 \sigma$ & $1.735(13) \times 10^{-5} \mathrm{~Hz} /\left(E_{\mathrm{r}} \mathrm{MHz}\right)$ & {$[31]$} \\
\hline Magic frequency near $390 \mathrm{~nm}$ & $0.001 \sigma$ & $2 \times 10^{-4} \sigma$ & $-0.04 \sigma$ & $766695 \mathrm{GHz}$ & 768917(18) GHz & [30] \\
\hline Tune-out frequency $v_{\text {to }}$ near $689 \mathrm{~nm}$ & $0.04 \sigma$ & $-0.009 \sigma$ & $434927 \mathrm{GHz}$ & $-0.009 \sigma$ & $434972.130(10) \mathrm{GHz}$ & [32] \\
\hline$\alpha\left(5 s 5 p{ }^{3} P_{0}, v=v_{\text {to }}\right)$ & $1.07 \sigma$ & $0.64 \sigma$ & $2.37 \times 10^{-38} \mathrm{C} \mathrm{m}^{2} / \mathrm{V}$ & $0.64 \sigma$ & $2.564(13) \times 10^{-38} \mathrm{C} \mathrm{m}^{2} / \mathrm{V}$ & {$[32]$} \\
\hline $5 s^{2}{ }^{1} S_{0}$ Thomas-Reiche-Kuhn sum & $5.1 \sigma$ & 2.5 & $0.95 \sigma$ & 2.5 & $2.0(1)$ & {$[36]$} \\
\hline $5 s 5 p{ }^{3} P_{0}$ Thomas-Reiche-Kuhn sum & $1.3 \sigma$ & 2.2 & $-2.54 \sigma$ & 2.2 & $2.0(1)$ & [36] \\
\hline Light shift at clock transition & $-26.2 \mathrm{~Hz} \mathrm{~cm}^{2} / \mathrm{W}$ & $-26.2 \mathrm{~Hz} \mathrm{~cm}^{2} / \mathrm{W}$ & $-24.1 \mathrm{~Hz} \mathrm{~cm}^{2} / \mathrm{W}$ & $-26.3 \mathrm{~Hz} \mathrm{~cm}^{2} / \mathrm{W}$ & & \\
\hline
\end{tabular}


The radius of convergence $r$ of a power series

$$
f(z)=\sum_{k=0}^{\infty} a_{k} z^{k}
$$

can be calculated by the ratio test

$$
r=\lim _{k \rightarrow \infty}\left|\frac{a_{k}}{a_{k+1}}\right| .
$$

Using the asymptotic behavior of the Bernoulli numbers [44], Eq. 24.11.1,

$$
B_{2 k} \sim \frac{(-1)^{k+1} 2(2 k) !}{(2 \pi)^{2 k}},
$$

we thus obtain

$$
r=\lim _{k \rightarrow \infty}\left|\frac{a_{k}}{a_{k+1}}\right|=\lim _{k \rightarrow \infty} \frac{1}{2 k(2 k+1)}=0 .
$$

\section{APPENDIX B: FIT RESULTS}

Adjusted parameters and output data of the fits discussed in the main text are summarized in Table IV. Fit results are reported as a deviation from the literature value in units of its uncertainty $\sigma$ for quantities that are used to constrain the fit procedure. Derived atomic properties and Einstein coefficients $A$ that are adjusted without direct constraints are reported as their numerical values.

Fit 1 uses all data discussed in the main text in the fit. Fit 2 does not use the Thomas-Reiche-Kuhn sum rule. Fit 3 excludes tune-out data from [32]. Fit 4 uses only fit constraints at low transition energies and therefore drops the blue magic wavelength and Thomas-Reiche-Kuhn sum rule as constraints.
[1] C. Sanner, N. Huntemann, R. Lange, C. Tamm, E. Peik, M. S. Safronova, and S. G. Porsev, Optical clock comparison test of Lorentz symmetry, Nature (London) 567, 204 (2019).

[2] W. F. McGrew, X. Zhang, H. Leopardi, R. J. Fasano, D. Nicolodi, K. Beloy, J. Yao, J. A. Sherman, S. A. Schäffer, J. Savory, R. C. Brown, S. Römisch, C. W. Oates, T. E. Parker, T. M. Fortier, and A. D. Ludlow, Towards the optical second: Verifying optical clocks at the SI limit, Optica 6, 448 (2019).

[3] S. M. Brewer, J.-S. Chen, A. M. Hankin, E. R. Clements, C. W. Chou, D. J. Wineland, D. B. Hume, and D. R. Leibrandt, ${ }^{27} \mathrm{Al}^{+}$ Quantum-Logic Clock with a Systematic Uncertainty below $10^{-18}$, Phys. Rev. Lett. 123, 033201 (2019).

[4] T. Bothwell, D. Kedar, E. Oelker, J. M. Robinson, S. L. Bromley, W. L. Tew, J. Ye, and C. J. Kennedy, JILA SrI optical lattice clock with uncertainty of $2.0 \times 10^{-18}$, Metrologia 56, 065004 (2019).

[5] S. G. Porsev and A. Derevianko, Multipolar theory of blackbody radiation shift of atomic energy levels and its implications for optical lattice clocks, Phys. Rev. A 74, 020502(R) (2006).

[6] F. Riehle, Towards a redefinition of the second based on optical atomic clocks, C. R. Phys. 16, 506 (2015), special issue on The measurement of time/La mesure du temps.

[7] W. M. Itano, L. L. Lewis, and D. J. Wineland, Shift of ${ }^{2} S_{1 / 2}$ hyperfine splittings due to blackbody radiation, Phys. Rev. A 25, 1233 (1982).

[8] A. Bauch and R. Schröder, Experimental Verification of the Shift of the Cesium Hyperfine Transition Frequency due to Blackbody Radiation, Phys. Rev. Lett. 78, 622 (1997).

[9] T. Middelmann, S. Falke, C. Lisdat, and U. Sterr, High Accuracy Correction of Blackbody Radiation Shift in an Optical Lattice Clock, Phys. Rev. Lett. 109, 263004 (2012).

[10] M. S. Safronova, S. G. Porsev, U. I. Safronova, M. G. Kozlov, and C. W. Clark, Blackbody radiation shift in the Sr optical atomic clock, Phys. Rev. A 87, 012509 (2013).

[11] T. L. Nicholson, S. L. Campbell, R. B. Hutson, G. E. Marti, B. J. Bloom, R. L. McNally, W. Zhang, M. D. Barrett, M. S. Safronova, G. F. Strouse, W. L. Tew, and J. Ye, Systematic evaluation of an atomic clock at $2 \times 10^{-18}$ total uncertainty, Nat. Commun. 6, 6896 (2015).
[12] R. Hobson, W. Bowden, A. Silva, C. F. A. Baynham, H. S. Margolis, P. E. G. Baird, P. Gill, and I. R. Hill, A strontium optical lattice clock with $1 \times 10^{-17}$ uncertainty and measurement of its absolute frequency, Metrologia 57, 065026 (2020).

[13] I. Ushijima, M. Takamoto, M. Das, T. Ohkubo, and H. Katori, Cryogenic optical lattice clocks, Nat. Photon. 9, 185 (2015).

[14] M. Takamoto, I. Ushijima, N. Ohmae, T. Yahagi, K. Kokado, H. Shinkai, and H. Katori, Test of general relativity by a pair of transportable optical lattice clocks, Nat. Photon. 14, 411 (2020).

[15] J. A. Sherman, N. D. Lemke, N. Hinkley, M. Pizzocaro, R. W. Fox, A. D. Ludlow, and C. W. Oates, High Accuracy Measure of Atomic Polarizability in an Optical Lattice Clock, Phys. Rev. Lett. 108, 153002 (2012).

[16] K. Beloy, J. A. Sherman, N. D. Lemke, N. Hinkley, C. W. Oates, and A. D. Ludlow, Determination of the $5 d 6 s^{3} D_{1}$ state lifetime and blackbody-radiation clock shift in Yb, Phys. Rev. A 86, 051404(R) (2012).

[17] J. W. Farley and W. H. Wing, Accurate calculation of dynamic Stark shifts and depopulation rates of Rydberg energy levels induced by blackbody radiation. Hydrogen, helium, and alkalimetal atoms, Phys. Rev. A 23, 2397 (1981).

[18] C. Lisdat, S. Dörscher, I. Nosske, and U. Sterr, Open dataset.

[19] M. Yasuda, T. Kishimoto, M. Takamoto, and H. Katori, Photoassociation spectroscopy of ${ }^{88} \mathrm{Sr}$ : Reconstruction of the wave function near the last node, Phys. Rev. A 73, 011403(R) (2006).

[20] J. E. Sansonetti and G. Nave, Wavelengths, transition probabilities, and energy levels for the spectrum of neutral strontium (Sr I), J. Phys. Chem. Ref. Data 39, 033103 (2010).

[21] G. von Oppen, Doppelresonanzuntersuchung des Einflusses eines elektrischen Feldes auf die Zeeman-Aufspaltung des $5 s 5 p^{3} P_{1}$-Terms im Sr $I$-Spektrum, Z. Phys. 227, 207 (1969).

[22] H. Bethe and E. Salpeter, Quantum mechanics of one- and twoelectron systems, in Handbuch der Physik, edited by S. Flügge (Springer, Berlin, 1957), Vol. XXXV.

[23] Yu. Ralchenko, A. E. Kramida, J Reader, NIST Atomic Spectra Database (version 4), http://www.nist.gov/pml/data/asd.cfm. 
[24] H. L. Schwartz, T. M. Miller, and B. Bederson, Measurement of the static electric dipole polarizabilities of barium and strontium, Phys. Rev. A 10, 1924 (1974).

[25] B. K. Sahoo and B. P. Das, Relativistic coupled-cluster studies of dipole polarizabilities in closed-shell atoms, Phys. Rev. A 77, 062516 (2008).

[26] Y. Singh, B. K. Sahoo, and B. P. Das, Correlation trends in the ground-state static electric dipole polarizabilities of closed-shell atoms and ions, Phys. Rev. A 88, 062504 (2013).

[27] P. Schwerdtfeger and J. K. Nagle, 2018 table of static dipole polarizabilities of the neutral elements in the periodic table, Mol. Phys. 117, 1200 (2019).

[28] C. Shi, J.-L. Robyr, U. Eismann, M. Zawada, L. Lorini, R. Le Targat, and J. Lodewyck, Polarizabilities of the ${ }^{87} \mathrm{Sr}$ clock transition, Phys. Rev. A 92, 012516 (2015).

[29] R. Le Targat, L. Lorini, Y. Le Coq, M. Zawada, J. Guéna, M. Abgrall, M. Gurov, P. Rosenbusch, D. G. Rovera, B. Nagórny, R. Gartman, P. G. Westergaard, M. E. Tobar, M. Lours, G. Santarelli, A. Clairon, S. Bize, P. Laurent, P. Lemonde, and J. Lodewyck, Experimental realization of an optical second with strontium lattice clocks, Nat. Commun. 4, 2109 (2013).

[30] M. Takamoto, H. Katori, S. I. Marmo, V. D. Ovsiannikov, and V. G. Pal'chikov, Prospects for Optical Clocks with a Blue-Detuned Lattice, Phys. Rev. Lett. 102, 063002 (2009).

[31] I. Ushijima, M. Takamoto, and H. Katori, Operational Magic Intensity for Sr Optical Lattice Clocks, Phys. Rev. Lett. 121, 263202 (2018).

[32] A. Heinz, A. J. Park, N. Šantić, J. Trautmann, S. G. Porsev, M. S. Safronova, I. Bloch, and S. Blatt, State-Dependent Optical Lattices for the Strontium Optical Qubit, Phys. Rev. Lett. 124, 203201 (2020).

[33] X. Baillard, M. Fouché, R. Le Targat, P. G. Westergaard, A. Lecallier, Y. Le Coq, G. D. Rovera, S. Bize, and P. Lemonde, Accuracy evaluation of an optical lattice clock with bosonic atoms, Opt. Lett. 32, 1812 (2007).
[34] T. Akatsuka, M. Takamoto, and H. Katori, Optical lattice clocks with non-interacting bosons and fermions, Nat. Phys. 4, 954 (2008).

[35] Q. Xu, X. Lu, J. Xia, Y. Wang, and H. Chang, Measurement of the probe Stark shift of the ${ }^{87} \mathrm{Sr}$ optical lattice clock using the frequency modulation spectroscopy, Appl. Phys. Lett. 119, 101105 (2021).

[36] E. U. Condon and G. H. Shortley, The Theory of Atomic Spectra (Cambridge University Press, Cambridge, UK, 1953).

[37] G. García and J. Campos, Transition probabilities for triplet levels of Sr(I), J. Quant. Spectrosc. Radiat. Transfer 39, 477 (1988).

[38] G. Jönsson, C. Levinson, A. Persson, and C. G. Wahlström, Natural radiative lifetimes in the ${ }^{1} P_{1}$ and ${ }^{1} F_{3}$ sequences of $\mathrm{Sr}$ I, Z. Phys. A 316, 255 (1984).

[39] H. G. C. Werij, C. H. Greene, C. E. Theodosiou, and A. Gallagher, Oscillator strengths and radiative branching ratios in atomic Sr, Phys. Rev. A 46, 1248 (1992).

[40] F. M. Kelly and M. S. Mathur, Lifetimes of some triplet levels of neutral strontium, Can. J. Phys. 54, 800 (1976).

[41] S. G. Porsev, A. D. Ludlow, M. M. Boyd, and J. Ye, Determination of $\mathrm{Sr}$ properties for a high-accuracy optical clock, Phys. Rev. A 78, 032508 (2008).

[42] GUM, Guide to the expression of uncertainty in measurement, ISO/TAG 4 (ISO, 1993, corrected and reprinted, 1995, in the name of the BIPM, IEC, IFCC, ISO, UPAC, IUPAP and OIML (1995), available at www.bipm.org/en/committees/ jc/jcgm/publications.

[43] A. J. Park, J. Trautmann, N. Šantić, V. Klüsener, A. Heinz, I. Bloch, and S. Blatt, Cavity-enhanced optical lattices for scaling neutral atom quantum technologies, arXiv:2110.08073.

[44] NIST Digital Library of Mathematical Functions, edited by F. W. J. Olver, A. B. Olde Daalhuis, D. W. Lozier, B. I. Schneider, R. F. Boisvert, C. W. Clark, B. R. Miller, B. V. Saunders, H. S. Cohl, and M. A. McClain (Cambridge University Press, Cambridge, UK, 2010). 